\title{
THE IMPORTANCE OF BRONCHOSCOPY IN THE RECOGNITION OF NON-OPAQUE FOREIGN BODY IN INFANTS AND CHILDREN
}

\author{
BY \\ N. ASHERSON, M.A., M.B., B.S., F.R.C.S.
}

Surgeon to the Central London Throat, Nose and Ear Hospital and to the Ear, Nose and Throat Department of the Queen's Hospital for Children ; Consulting Surgeon to the London County Council.

When the presence of a non-opaque foreign body in a main or lower lobe bronchus is suspected, the following radiographs will be required, in addition to screening in order to note the positive movements of the mediastinum, heart and diaphragm : (1) A postero-anterior view at the end of inspiration ; (2) a postero-anterior view at the end of expiration; (3) a lateral view; and (4) occasionally a bronchogram.

Radiographs of the normal chest when in full inspiration and full expiration show that the following points can be noted : (1) An increased translucency of both lungs during inspiration ; (2) the position of the heart, mediastinum and the trachea is unaltered and in the mid-line ; (3) the dome of the diaphragm is depressed with inspiration and rises with expiration; (4) the intercostal spaces are widened with inspiration ; (5) the apices of the lungs are contracted in inspiration ; and (6) a decreased translucency of the lungs in expiration. When one lung is in the condition of 'obstructive emphysema,' the affected lung remains hypertranslucent at the end of expiration, due to the trapped air being unable to escape, and in a suspected case of a non-opaque foreign body radiographs in both inspiration and expiration are essential.

\section{Case reports}

The following case records emphasize the diagnostic and therapeutic value of bronchoscopy in suitable cases.

Case 1.-Non-opaque foreign body (pea-nut kernel) blocking the left main bronchus ; bronchoscopy with clearing of the bronchus ; subsequent sojourn of portion of a pea-nut for over twelve months in the left lower bronchus, with no symptoms pointing to its presence, except persistent localized physical signs at the left base ; removal of a pea-nut by bronchoscopy twelve months after inspiration; recovery.

H. L., aged five years, was admitted in September, 1937, with dyspnoea following the 'swallowing' of a pea-nut kernel twenty-four hours before. 
The child was flushed, not cyanosed, with a hoarse voice and a slight dry cough. Temperature $101^{\circ} \mathrm{F}$., respirations 32 . Movements were diminished over the left chest, especially at the base. A pleural rub was heard over the left chest in front. Radiographs of the chest taken at the end of inspiration and expiration showed a greatly diminished air entry to the left lung (see fig. 1, A and B).
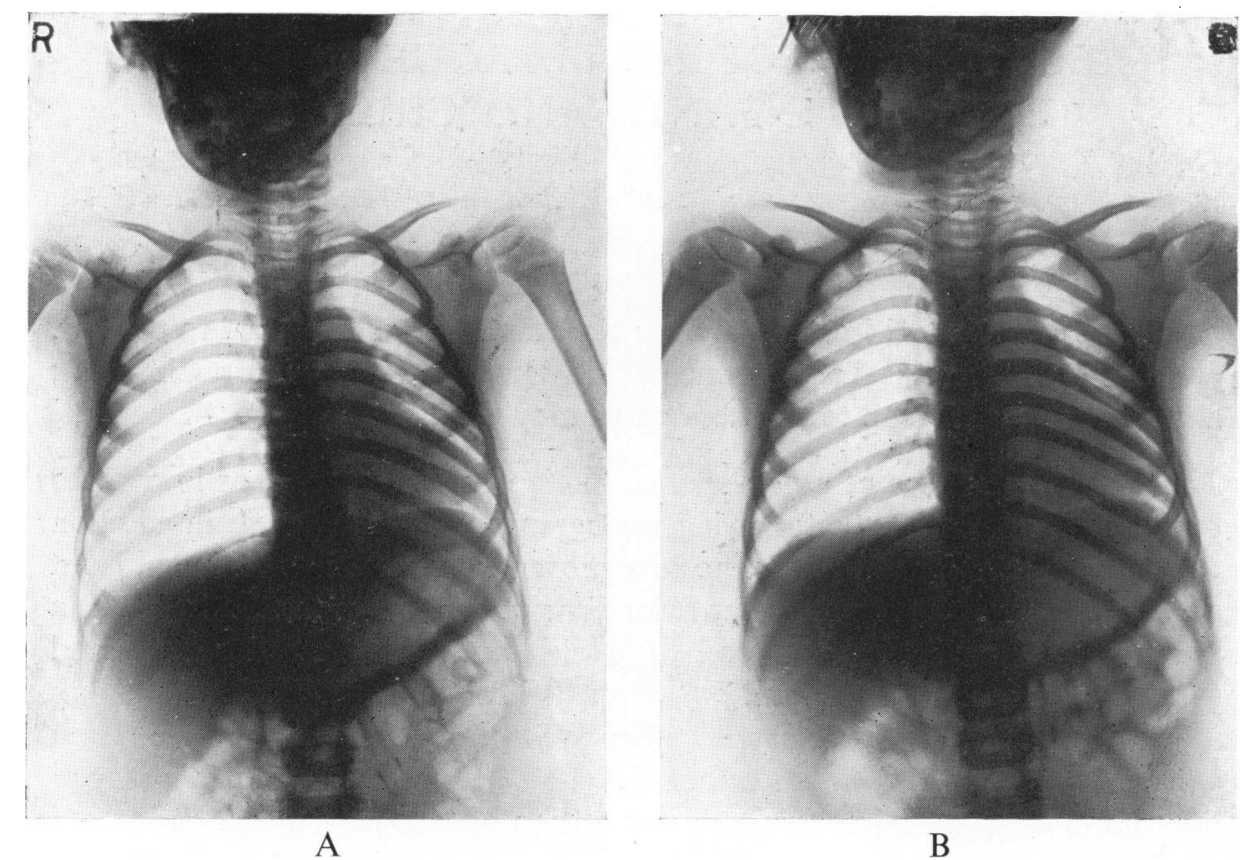

FIG. 1, A and B. (Case 1.) Pea-nut in left main bronchus producing obstructive atelectasis. Radiographs at the end of inspiration $(A)$ and end of expiration $(B)$ before bronchoscopy, September 4, 1937.

The heart and mediastinum were pulled over to the left, so that the right border of the vertebral column was exposed. The left dome of the diaphragm was raised, and remains so in full inspiration. The heart, trachea and mediastinum shadow were drawn over more to the left on inspiration and receded slightly to the right on expiration when the right border of the heart became visible at the right border of the vertebral column. The aeration of the left lung was not altered at the end of inspiration and of expiration. These radiological signs were caused by a left-sided atelectasis.

The same radiological picture is also consistent with 'obstructive emphysema' of the right lung, a condition due to impaction of a foreign body in the right main bronchus causing a ball-valve obstruction in which air can pass into but cannot pass out of the obstructed bronchus. The lung tissue becomes hypertranslucent at the end of the inspiration as well as at the end of the expiration. However, in this case the foreign body was found in the left bronchus.

Bronchoscopy was performed on September 4, 1937, under chloroform anaesthesia, using the 5-mm. Jackson instrument. The left main bronchus was cleared of mucus, but no foreign body was seen.

SUBSEQUENT COURSE.-Within three days the temperature became normal ; the breathing was good ; rhonchi were heard all over the chest; air entry was equal on both sides; the heart reverted to its normal position. A skiagraph 
on September 13,1937, showed the lungs filled with air equally and the diaphragm was normal (fig. 2, A and B).

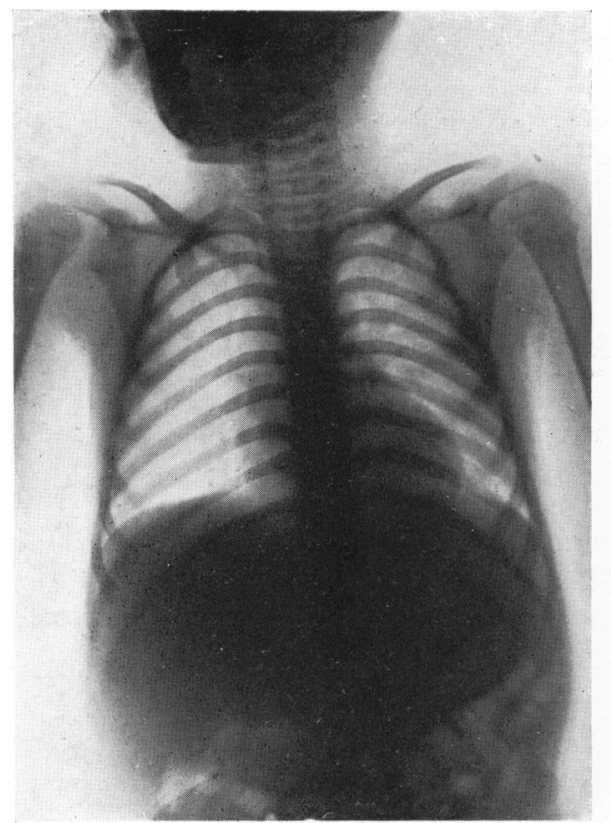

A

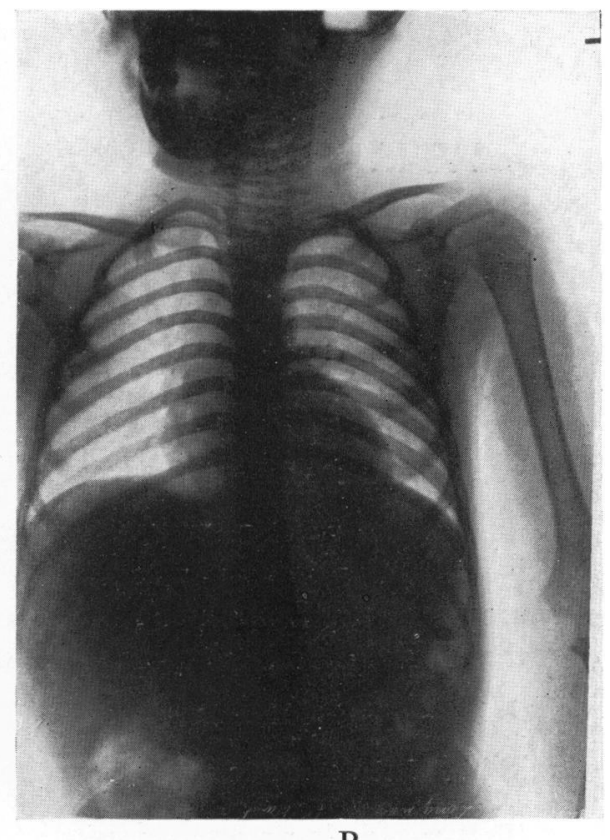

B

FIG. 2, A and B. (Case 1.) After bronchoscopy ; lungs clear. Similar pictures were obtained on January 3, 1938, and September 19, 1938.

LATER PROGRESS.-The child remained well, with occasional bouts of coughing, but no other symptoms, and if a routine three-monthly examination of the chest had not been ordered the child would not have been brought for advice. Radiographs on January 3, 1938, and September 19, 1938, showed no abnormality. On each occasion on which the chest was examined at the threemonthly routine visit a slightly diminished air entry was detected at the left base, with a sonorous expiratory rhonchus persistently localized to an area the size of a shilling, and sounding like an expiratory squeak. The child was admitted for observation, and during five days in hospital the temperature was normal and there was no coughing. Radiographs were negative. On deep inspiration and expiration, coarse râles and crepitations could be heard at the base.

On September 22, 1938, under chloroform anaesthesia, using the Negus infant instrument, bronchoscopy was repeated. The trachea and right main bronchus were normal. The left main bronchus was normal, but at its depth a pool of yellow pus was observed, filling the left lower lobe bronchus. This pus was aspirated, and a portion of pea-nut became visible. It was removed in fragments by a forward-grasping forceps, with the use of a sucker in between. The nut was friable and fragmented easily. The bronchus was finally cleared. The macerated portions removed are shown diagrammatically in fig. 3. A piece of nut was found at the end of the aspirating tube, and many small pieces in the receptacle of the sucker. The time taken was twenty minutes. Within one hour of the bronchoscopy the left base had a normal air entry and percussion note, and the expiratory squeak had disappeared. In June, 1939, the child was still well and the left base clear. 
Comment.-This case illustrated that persistent signs at the base of a lung should lead to exploratory bronchoscopy. In this case part of a pea-nut had remained in a lower lobe bronchus for over a year with little or no symptoms and only slight signs. It also indicates the necessity for repeated routine exami-

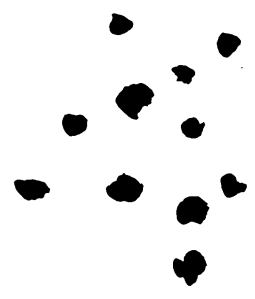

1

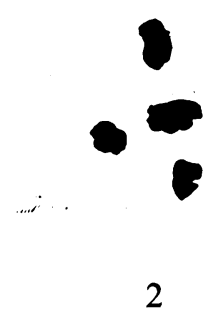

2

FIG. 3.-Portions of the macerated pea-nut-part 1 removed by the aspirating tube and rescued from the sucker bottle ; part 2 removed by the forceps.

nations of the chest after bronchoscopy for a suspected non-opaque foreign body, when that body has not been completely removed. Failure to locate it by bronchoscopy is no final evidence of its absence, unless this is substantiated by the clinical examination of the chest at subsequent regular intervals.

Case 2.-Non-opaque foreign body (piece of egg-shell), blocking the right main bronchus of an infant ; bronchoscopy ; recovery.

T. S., aged eleven months, was admitted on June 20,1938 , with the history that five days previously he was playing with some broken egg-shells in his pram. His breathing was noticed to become suddenly distressed and when picked up he started a hacking cough which went on for five minutes. The infant did not go red in the face, was well for an hour, and then started another bout of coughing. He became distressed at night and was thought to have pneumonia. He was better during the day and relapsed at night. The respiration became noisy and asthma was then diagnosed. The noisy respiration persisted.

On admission he showed noisy and distressed respiration. Temperature $102^{\circ} \mathrm{F}$., pulse 162 , respiration 64 . There was apparent laryngeal obstruction, with recession of the intercostal spaces on inspiration and excessive abdominal breathing, but no cyanosis. Dullness elicited over the entire right side of the chest with diminished air entry at the right base suggested massive collapse of the right lung. Skiagraphs of the chest (fig. 4) showed the heart and mediastinum drawn over to the right and the diaphragm raised on the right with collapse of the right upper and middle lobes.

On June 21, 1938, under chloroform anaesthesia preceded by seconal bronchoscopy was performed using the Negus suckling instrument. Pus welled up through the glottis and was sucked away ; the trachea also contained pus. Aspiration revealed an acute congestive laryngitis, tracheitis and bronchitis. The right main bronchus was filled with pus which was aspirated and the lumen cleared. No foreign body was actually observed. Immediately after bronchoscopy, which lasted twenty minutes, the breathing was easier and the air entry was equal on both sides. A relapse occurred after return to the ward, and the respiratory embarrassment returned till the next day, when improvement followed vomiting. Respirations 40 , temperature $103^{\circ} \mathrm{F}$., pulse 92 . June 23 , temperature $99^{\circ}$; respirations 30 . June 24 , the child was convalescent ; skiagraphs showed equal air entry (fig. 5). 


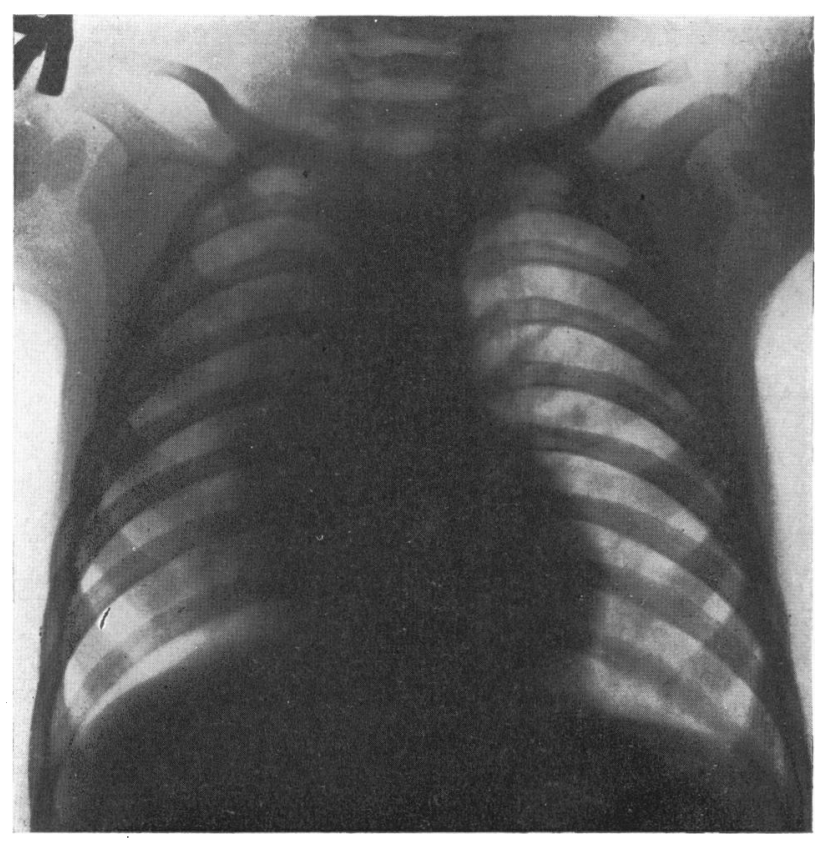

Fig. 4. (Case 2.) Before bronchoscopy.

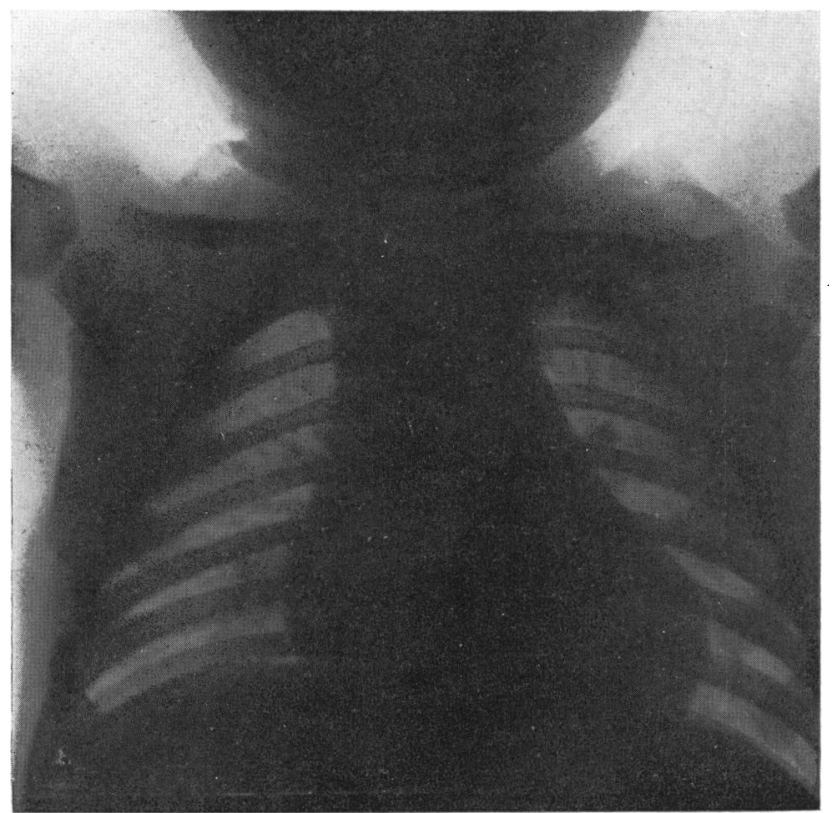

F G. 5. (Case 2.) After bronchoscopy. 
Case 3.-Unsuspected non-opaque foreign body (pea-nut) in the right bronchus for over three months producing relapsing collapse of the right lower lobe ; removal of pea-nut at bronchoscopy ; recovery.

G. C., aged $6 \frac{1}{2}$ years, was admitted on December 29,1937 , with a history of a cough for three weeks, worse for the last four days. On admission the temperature was $101 \cdot 6^{\circ}$ F., pulse 136 , respirations normal. Breath sounds were faint and there was an impaired percussion note at the right base.

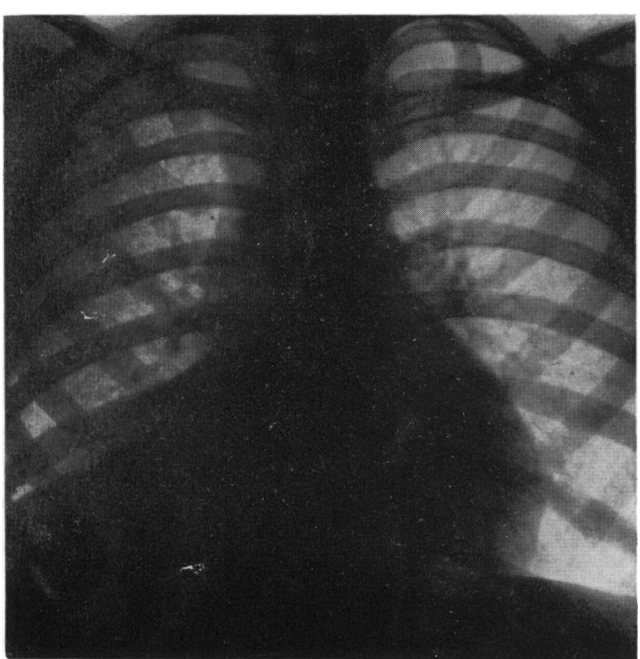

Fig. 6. (Case 3.) On admission, December 30, 1937, showing triangular shadows due to collapse of lower lobe of right lung.

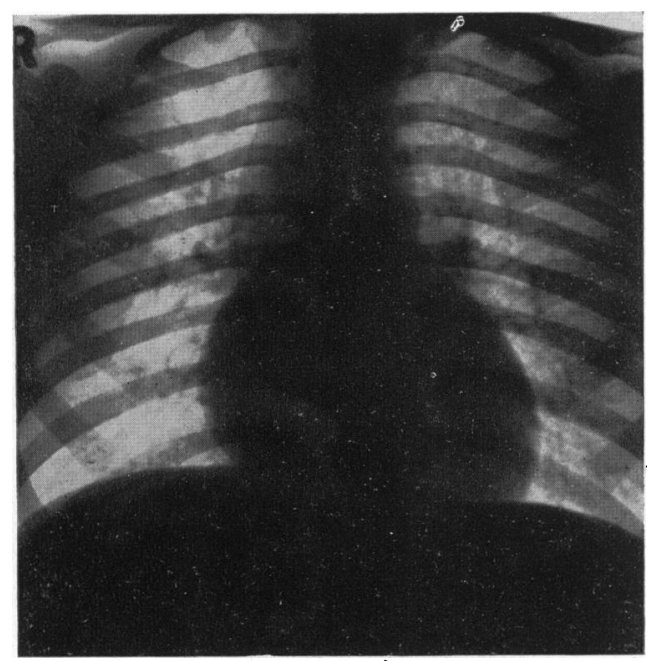

Fig. 7. (Case 3.) Jan. 6, 1938.

A radiograph (fig. 6) showed the typical triangular shadows produced by collapse of the lower lobe of the right lung. The right diaphragm was raised on screening. Mantoux test negative. The temperature settled in five days ; there was no cough. 
A radiograph on January 6, 1938 (fig. 7) showed the chest clear, and as there were no abnormal physical signs he was sent home.

Re-admitted on February 18, 1938, the child had been well until seven days before, when he became feverish. Cough which had been present for three

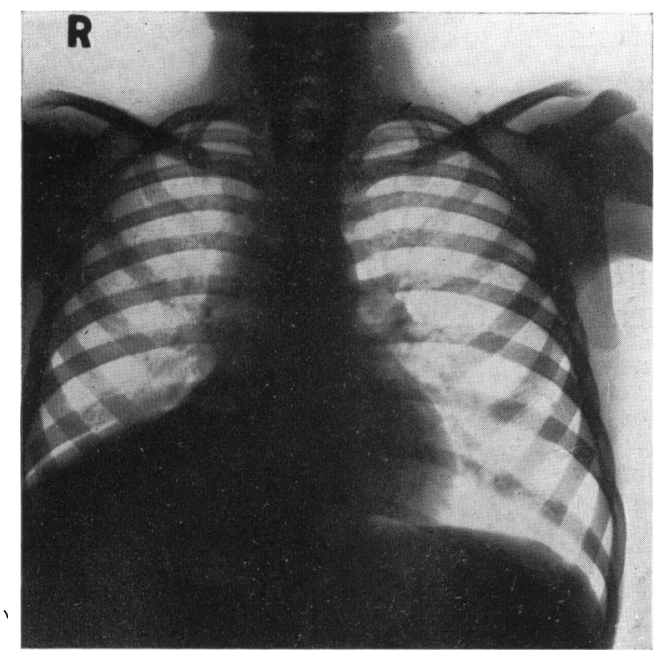

FIG. 8. (Case 3.) Before bronchoscopy, on readmission, February 21, 1938, still shows shadows due to collapse of middle and lower lobes of right lung.

weeks was severe for the past week, with much sputum. The right base showed diminished movement and poor breath-sounds and impaired percussion note.

A skiagraph on February 21, 1938 (fig. 8), again showed collapse of right middle and lower lobes. A diagnosis was made of recurrent collapse due either to enlarged mediastinal glands or to intrabronchial obstruction. Tuberculosis

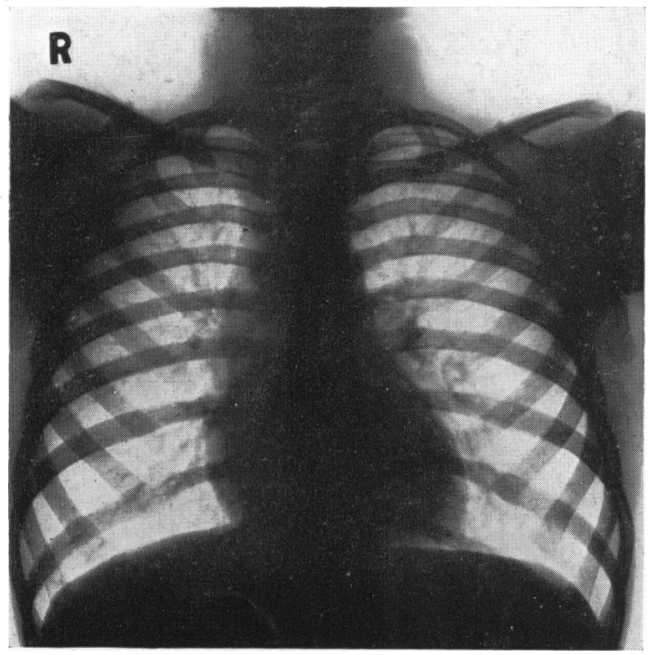

Fig. 9. (Case 3.) After bronchoscopy.

was excluded by a negative Mantoux test. A bronchogram revealed an obstruction in the right main bronchus just beyond the bifurcation. 
On March 31, 1938, under chloroform anaesthesia a bronchoscopy was performed (using the child's Negus instrument) and a pea-nut kernel was removed intact from the right lower lobe bronchus. The pea-nut was unchanged by its prolonged sojourn in the bronchus. The time taken was five minutes ; recovery was complete.

This patient was under the care of Dr. Sidney Owen.

Comment.-The skiagraphs taken at intervals show the collapse and reexpansion of the right middle and lower lobes. The variations in the x-ray findings show that repeated clinical and x-ray examinations are necessary to enable a competent opinion to be given, as in Case 1. There was no history of an inhaled foreign body in this case. A skiagraph on April 2, 1938 (fig. 9), showed no abnormality and there were abnormal physical signs in the chest. 